\title{
Correction
}

\section{Correction: Takata et al., Astrocyte Calcium Signaling Transforms Cholinergic Modulation to Cortical Plasticity In Vivo}

In the article "Astrocyte Calcium Signaling Transforms Cholinergic Modulation to Cortical Plasticity In Vivo" by Norio Takata, Tsuneko Mishima, Chihiro Hisatsune, Terumi Nagai, Etsuko Ebisui, Katsuhiko Mikoshiba, and Hajime Hirase, which appeared on pages 18155-18165 of the December 7, 2011 issue, the authors regret an error in the single-train NBM stimulation parameter, described in the Materials and Methods section. The correct parameter is: 50 pulses ( $200 \mu \mathrm{A}, 0.5 \mathrm{~ms}, 100 \mathrm{~Hz})$. The parameter described in the Results section is correct.

Doi: 10.1523/JNEUROSCI.2659-12.2012 